\title{
DESIGN ISSUES OF ROUTING PROTOCOLS FOR MANET'S
}

\author{
Harikrishna $T^{1}$, Dr Subramanyam.A ${ }^{2}$ \\ ${ }^{1}$ Research Scholar in Rayalaseema University, Kurnool, \\ ${ }^{2}$ Professor \& Dean, Annamacharya Institute of Technology and Sciences, Rajampet
}

\begin{abstract}
Mobile Ad hoc Network (MANET) is a set of wireless mobile nodes dynamically forming a temporary network. The goal of the architecture is to provide communication facilities between end-users without any centralized infrastructure. In such a network, each mobile node operates not only as a host but also as a router. This paper studies diverse routing protocols, issues and requirements comparatively in MANET routing and design considerations including classifications based on layers and others. Keywords: Mobile Ad hoc Networks, Wireless Routing, Routing Protocol, Wireless Routing.
\end{abstract}

\section{I.INTRODUCTION}

Mobile Ad hoc Network is a set of mobile nodes that are connected by wireless links. The goal of this architecture is to provide communication facilities between end-users without any centralized infrastructure. In such a network, each mobile node operates not only as a host but also as a router. MANET's, bear great application potential in dynamic scenarios including disaster and emergency relief, mobile conferencing, sensor dust, battle field communication, and so on. There are some challenges in designing mobile ad hoc network. The first of these is that all nodes in MANET might be mobile, including the corresponding destinations, the source nodes, and the routing nodes forward traffic between nodes. A break may occur in the link due to limited range of wireless transmission. Another reason is lack of administration. The functions like determining network topology, multiple accesses, and data routing over suitable multi hop paths, should be implemented $\mathrm{n}$ a distributed way. The designing challenges are met by providing solutions at different layers of network. The physical layer deals with fading, path loss and multi-user interference order to sustain stabilized communication links among the nodes. The data link layer should create reliability to the physical link as well as resolve the contention among unsynchronized users via the transmission of packets on a shared channel.

II.ISSUES OF ROUTING IN MANET'S

Many challenges are in front of wireless ad-hoc networks [6], some of the key issues to be considered for routing are,

\subsection{Asymmetric links}

Most of the wired networks rely on the symmetric links which are always fixed. But this is not a case with ad-hoc networks as the nodes are Mobile and constantly changing their position within network. But this does not tell anything about the quality of the connection in the reverse direction.

\subsection{Routing Overhead}

In wireless ad hoc networks, nodes often change their location within network. So, some stale routes are generated in the routing table which leads to unnecessary routing overhead.

\subsection{Bandwidth constraint}

Since the channel is shared by all nodes in the network domain, the bandwidth available per wireless link depends on the number of nodes and the traffic they handle. Thus only a friction of the total bandwidth is available for every node.

\subsection{Interference}

This is the major problem with mobile ad-hoc networks as links come and go depending on the transmission characteristics, one transmission might interfere with another one and node might overhear transmissions of other nodes and can corrupt the total transmission.

\subsection{Location dependent contention}

The load on the wireless channel varies with the number of nodes present in a given geographical region. This makes the contention for the channel high when the number of nodes increases. The high contention for the channel results in a high number of collisions and subsequent networks, routing tables must somehow reflect these Changes in topology and routing algorithms 2.6 Lack of Administration

In MANET all the functions of network like network topology's determination, multiple accesses, data routing over suitable multi hop.

2.7 Wastage of bandwidth

A good routing protocol should have build-in mechanisms for distributing the network load uniformly across the network so that the formation of regions where channel contention is high can be avoided.

\subsection{Dynamic Topology}

This is also the major problem with ad-hoc routing since the Topology is not constant. The mobile node might move or medium characteristics Might change. In ad-hoc paths should be implemented in a distributed way. Such tasks are challenging because the communication bandwidth is limited.

\subsection{Other Resource Constraint}

The constraints on resources such as computing power, battery capacity and buffer storage also limit the capability of a routing protocol.

2.10 Hidden Terminal Problem

It is the collision of packets at a receiving node due to the simultaneous transmission of those nodes that are not within the direct transmission range of the sender, but are within the transmission range of the receiver. Collision occurs when both nodes transmit packets at the same time without knowing about the transmission of each other. 


\subsection{Exposed Terminal Problem}

It is the inability of a node which is blocked due to transmission by a nearby transmitting node to transmit to another node.

III.REQUIREMENTS OF ROUTING PROTOCOL IN MANET'S

The major requirements of a routing protocol in Mobile Ad hoc Networks are as follows,

3.1 Quick Route reconfiguration

The unpredictable changes in the topology of the network require that the routing protocol be able to quickly perform route reconfiguration in order to handle path breaks and subsequent packet loss.

3.2 Minimum Route Acquisition Delay

The route acquisition delay for anode that does not have a route to a particular destination node should be as minimal as possible. This delay may vary with the size of the network and the network load.

\subsection{Loop-free Routing}

Due to the random movement of the nodes, transient loops may form in the route thus established. A routing protocol should dete $3 \mathrm{ct}$ such transient routing loops and take corrective actions.

3.4 Support for time-Oriented traffic

Tactical communications and similar applications require support for time-sensitive traffic. So the routing protocol should be able to support both hard real-time and soft real-time traffic.

\subsection{Minimum Control Overhead}

The control packets exchanged for finding a new route and maintaining existing routes should be kept as minimal as possible. The control packets consume precious bandwidth and can cause collisions with data packets, thereby reducing network throughput.

\subsection{Distributed Routing}

MANET is by characteristic a fully distributed wireless network and use of centralized routing is mere deteriorating the performance of routing. Hence the distributed routing is most required approach.

\subsection{Scalability}

It is the ability of a routing protocol performs efficiently in a network that can grow to large size. It requires minimization of control overhead and adaptation of the routing protocol to the network size.

\subsection{Provisioning of $Q o S$}

The goal of a routing protocol is to provide certain level of QoS by supporting differentiated classes of service.

3.9 Security

The routing protocol must be resilient to threats and vulnerabilities. It must have ability to avoid security threats like denial-of-service, impersonation and such other attacks.

\section{IV.ROUTING PROTOCOL PARAMETERS}

Parameter is a key element that must be considered in providing Quality of Service solution. The level of QoS and the associated QoS parameters also differ from application to application. The Table 1 gives the list of key QoS parameters required for different type of applications. The parameters to be considered for Routing in providing QoS provisioning are,

\subsection{Bandwidth}

It is the raw capability of a communications channel to move data through that channel. Typically measured in bits or bytes per second (or some, possibly large, multiple thereof). It refers to how wide the media is, not how fast. 4.2 Throughput

It is the amount of data that actuality moves from one point to another over a given amount of time. Many things effect throughput may include protocol, data loss, latency, and others.

Throughput=amount of data transferred / transmission time

\subsection{Delay or Latency}

It is the time elapsed from the departure of a data packet from the source node to the arrival at the destination node, including queuing delay, switching delay, propagation delay etc.,

Delay $=$ packet arrival time - packet start time

4.4 Jitter

It is a variation in packet delay at the receiver of the information. A network with constant latency has no variation (or jitter).

$$
\sum_{i=1}^{n}\left(\text { Delay }_{i}-\text { Delay }_{i-1}\right) / n-1
$$

4.5 Packet Loss

It occurs when one or more packets of data travelling across a computer network fail to reach their destination. Packet loss is typically caused by network congestion. Packet loss is measured as a percentage of packets lost with respect to packets sent.

\subsection{Scalability}

A system is considered scalable if it is capable of increasing its total output under an increased load when resources (typically hardware) are added.

\subsection{Security}

It is the protection from the unauthorized access and usage of the network resources

4.8 Availability

Availability of a system is typically measured as a factor of its reliability - as reliability increases, so does availability. IT IS THE RATIO OF (A) the total time a functional unit is capable of being used during a given interval to (b) the length of the interval.

\subsection{Energy Conservation}

It considers the reducing of energy consumption through using less energy for a constant service.

\subsection{Error rate}

The number of corrupted bits expressed as a percentage or fraction of the total sent.

\subsection{Packet Delivery Ratio (PDR)}

It is the measure of total number of packets successfully delivered to the destination.

PDR $=($ No. of packets delivered $/$ No. of packets sent) $* 100$

\begin{tabular}{l|l|}
\hline Application & Key QoS Parameters \\
\hline Multimedia Applications & Bandwidth, Delay etc., \\
\hline Military Applications & Security, Reliability, Delay, Bandwidth etc., \\
\hline $\begin{array}{l}\text { Emergency/Realtime Applications } \\
\text { Hybrid wireless network applications }\end{array}$ & $\begin{array}{l}\text { Availability, Reliability etc., } \\
\text { Maximum available link life, delay, } \\
\text { bandwidth, channel utilization etc., }\end{array}$ \\
\hline Sensor network applications & Battery life, Energy conservation etc., \\
\hline
\end{tabular}

Table 1: Applications and Key Parameters of QoS 


\section{V.CLASSIFICATION OF MANET ROUTING PROTOCOLS}

There are some criteria's for designing and classifying routing protocols such as the routing information is exchanged, Pre-compound routing, Periodical updates, Number of paths etc., There are several routing protocols proposed for wireless ad-hoc networks based on the above criteria's. The classification of protocols is shown in Figure 1. This classification of routing protocols can be made into three major categories,

\subsection{Proactive/Table Driven Routing Protocols}

In this every node maintains the network topology information in the form of routing tables by periodically exchanging routing information. Routing requires a path to a destination, it runs an appropriate path- finding algorithm on the topology information it maintains.

\subsection{Reactive or on-demand Routing Protocols}

It does not maintain the network topology information. They obtain the necessary path when it is required, by using a connection establishment process. Hence these protocols do not exchange routing information periodically.

\subsection{Hybrid Routing Protocols}

These combine the best features of the above two categories. Nodes within a certain distance from the node concerned or within a particular geographical region are set to be within the routing zone of the given mode. For routing within this zone a table driven approach is used. For nodes that are located beyond this zone on-demand approach is used.

\section{a. AD HOC ON-DEMAND DISTANCE VECTOR ROUTING} (AODV)

The Ad Hoc On-Demand Distance Vector routing protocol (AODV) is an improvement of the DestinationSequenced Distance Vector routing protocol (DSDV)[4] . DSDV has its efficiency in creating smaller ad-hoc networks. Since it requires periodic advertisement and global dissemination of connectivity information for correct operation, it leads to frequent system-wide broadcasts. Therefore the size of DSDV ad-hoc networks is strongly limited. When using DSDV, every mobile node also needs to maintain a complete list of routes for each destination within the mobile network. The advantage of AODV is that it tries to minimize the number of required broadcasts. It creates the routes on a on-demand basis, as opposed to maintain a complete list of routes for each destination. Therefore, the authors of AODV classify it as a pure on-demand route acquisition system

\section{b. DYNAMIC SOURCE ROUTING (DSR)}

The Dynamic Source Routing (DSR) protocol is an ondemand routing protocol based on source routing [3]. In the source routing technique, a sender determines the exact sequence of nodes through which to propagate a packet. The list of intermediate nodes for routing is explicitly contained in the packet's header. In DSR, every mobile node in the network needs to maintain a route cache where it caches source routes that it has learned. When a host wants to send a packet to some other host, it first checks its route cache for a source route to the destination. In the case a route is found, the sender uses this route to propagate the packet. Otherwise the source node initiates the route discovery process. Route discovery and route maintenance are the two major parts of the DSR protocol.

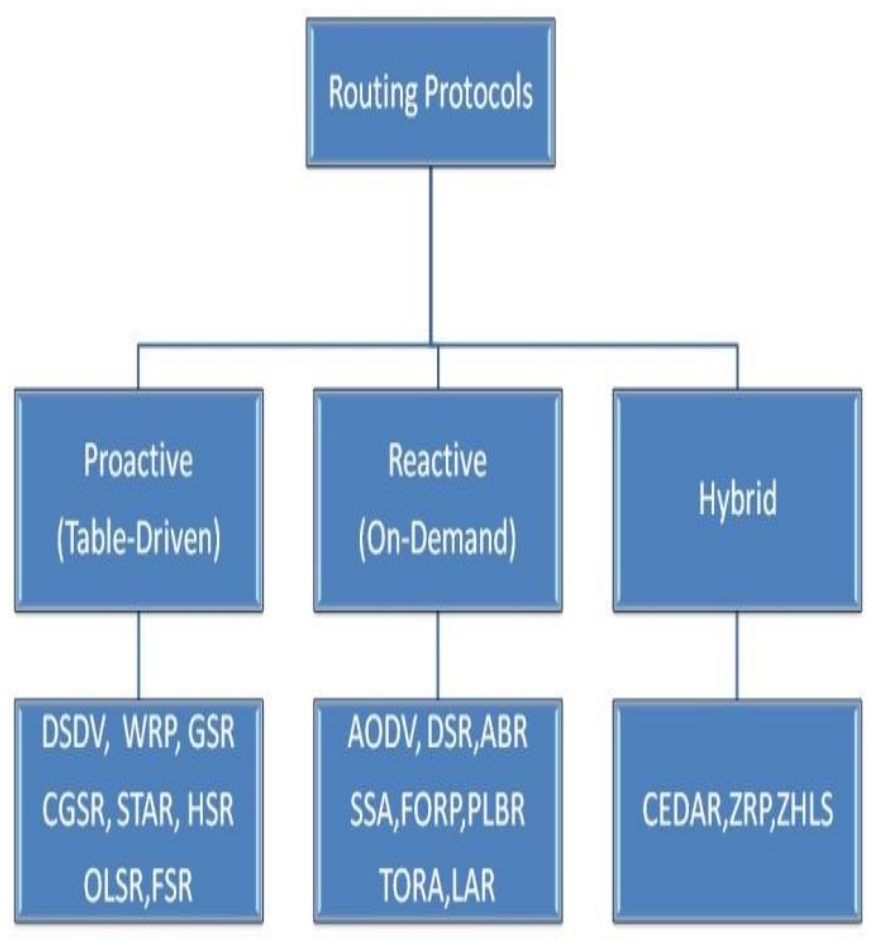

Figure 1 : Routing Protocols Classification [6]

\section{c. LOCATION-AIDED ROUTING (LAR)}

Ad hoc on-demand distance vector routing (AODV) and distance vector routing (DSR) that have been previously described are both based on different variations of flooding. The goal of Location-Aided Routing (LAR) described in [5] is to reduce the routing overhead by the use of location information. Position information will be used by LAR for restricting the flooding to a certain area [7]. In the LAR routing technique, route request and route reply packets similar to DSR and AODV are being proposed. The implementation in the simulator follows the LAR1 algorithm similar to DSR.

d. ZONE ROUTING PROTOCOL (ZRP)

In a mobile ad-hoc network, it can be assumed that most of the communication takes place between nodes close to each other. The Zone Routing Protocol (ZRP) described in [8] takes advantage of this fact and divides the entire network into overlapping zones of variable size. It uses proactive protocols for finding zone neighbors (instantly sending hello messages) as well as reactive protocols for routing purposes between different zones (a route is only established if needed). Each node may define its own zone size, whereby the zone size is defined as number of hops to the zone perimeter. For instance, the zone size may depend on signal strength, available power, reliability of different nodes etc. While ZRP is not a very distinct protocol, it provides a framework for other protocols. First of all, a node needs to discover its neighborhood in order to be able to build a zone and determine the perimeter nodes. In all perimeter nodes are printed in dark gray color - they build the border of A's zone with radius $\rho=$ 2. The detection process is usually accomplished by using 
the Neighbor Discovery Protocol (NDP). Every node periodically sends some hello messages to its neighbors. If it receives an answer, a point-to-point connection to this node exists. Nodes may be selected by different criteria be it signals strength, radio frequency, delay etc. The discovery messages are repeated from time to time to keep the map of the neighbors updated. The routing processes inside a zone are performed by the Intrazone Routing Protocol (IARP). This protocol is responsible for determine the routes to the peripheral nodes of a zone. It is Zone Routing Protocol (ZRP) generally a proactive protocol. Another type of protocol is used for the communication between different zones. It is called Interzone Routing Protocol (IERP) and is only responsible for routing between peripheral zones. A third protocol, the Border cast Resolution Protocol (BRP) is used to optimize the routing process between perimeter nodes. Thus, it is not necessary to flood all peripheral nodes, what makes queries become more efficient.

\section{e. INTRAZONE ROUTING PROTOCOL (IARP)}

The IARP protocol is used by a node to communicate with the other interior nodes of its zone. An important goal is to support unidirectional links, but not only symmetric links. It occurs very often, that a node A may send data to a node $\mathrm{B}$, but node $\mathrm{B}$ cannot reach node $\mathrm{A}$ due to interference or low transmission power for example. IARP is limited to the size of the zone $\rho$. The periodically broadcasted route discovery packets will be initialized with a Time To Live (TTL) field set to $\rho-1$. Every node which forwards the packet will now decrease this field by one until the perimeter is reached. In this case, the TTL field is 0 and the packet will be discarded. This makes sure that an IARP route request will never be forwarded out of a node's zone. As already mentioned, IARP is a proactive, table-driven protocol for the local neighborhood may change rapidly, and changes in the local topology are likely to have a bigger impact on a nodes routing behavior than a change on the other end of the network [9]. Proactive, table-driven routing delivers a fast, efficient search of routes to local hosts. Local routes are immediately available. Therefore, every node periodically needs to update the routing information inside the zone. Additionally, local route optimization is performed. This includes the following actions: - Removal of redundant routes - Shortening of routes, if a node can be reached with a smaller number of hops - Detecting of link failures and bypassing them through multiple local hops

\section{f. INTERZONE ROUTING PROTOCOL (IERP)}

The Interzone Routing Protocol is used to communicate between nodes of different zones. It is a reactive protocol and the route discovery process is only initiated on demand. This makes route finding slower, but the delay can be minimized by use of the Border cast Resolution Protocol. IERP takes advantage of the fact that IARP knows the local configuration of a zone. So a query is not submitted to all local nodes, but only to a node's peripheral nodes. Furthermore, a node does not send a query back to the nodes the request came from, even if they are peripheral nodes

\section{g. BORDER CAST RESOLUTION PROTOCOL (BRP)}

The Border cast Resolution Protocol is rather a packet delivery service than a full featured routing protocol [10]. It is used to send routing requests generated by IERP directly to peripheral nodes to increase efficiency. BRP takes advantage of the local map from IARP and creates a border cast tree of it. The BRP employs special query control mechanisms to steer route requests away from areas of the network that have already been covered by the query [10]. The use of this concept makes it much faster than flooding packets from node to node.

\section{h. OPTIMIZED LINK STATE ROUTING}

Unlike AODV and DSR, OLSR is a proactive link state protocol similar to OSPF, but with optimizations for ad hoc networks that reduce control traffic overhead and increase reactivity to topological changes. OLSR minimizes control traffic overhead in two ways. First, by using multi-point relays (MPRs) to transmit control messages through the network, second, by only requiring partial link state information to be flooded. OLSR relies on HELLO messages to maintain a neighbor set. In a HELLO message, a node announces its link set, neighbor set and MPR set. These messages only reach direct neighbors. In contrast to AODV, OLSR requires a symmetric link to establish connectivity with a neighbor. Actual link states are only propagated throughout the network by MPRs in Topology Control (TC) messages. TC messages contain sufficient link state to build the topology information base and to perform route calculation. Because of the proactive nature of OLSR, the protocol needs time to converge and reacts more slowly to topological changes.

\section{VI.COMPARISON OF ROUTING PROTOCOLS}

To facilitate a comparison among the different routing protocols according to the design constraints bandwidth/delay estimation, route discovery, resource reservation, route maintenance, route selection and network architecture. The Table. 1 gives the comparison of various routing protocols with these constraints.

6.1. Packet delivery ratio:

Packet delivery ratio is calculated by dividing the number of packets received by the destination through the number of packets originated by the application layer of the source (i.e. CBR source). It specifies the packet loss rate, which limits the maximum throughput of the network. The better the delivery ratio, the more complete and correct is the routing protocol.

\subsection{Routing overhead:}

The routing overhead describes how many routing packets for route discovery and route maintenance need to be sent in order to propagate the CBR packets. It is an important measure for the scalability of a protocol. It for instance determines, if a protocol will function in congested or low-bandwidth situations, or how much node battery power it consumes. If a protocol requires sending many routing packets, it will most likely cause congestion, collision and data delay in larger networks.

6.3. End-to-end delay:

End-to-end delay indicates how long it took for a packet to travel from the CBR source to the application layer of the destination. It represents the average data delay an application or a user experiences when transmitting data. 


\subsection{Hop count:}

Hop count is the number of hops a packet took to reach its destination.

\begin{tabular}{|c|c|c|c|c|c|c|c|}
\hline $\begin{array}{l}\text { Routing } \\
\text { Protocol }\end{array}$ & Parameter & $\begin{array}{l}\text { Bandwidth/ } \\
\text { Delay } \\
\text { Estimation }\end{array}$ & $\begin{array}{l}\text { Route } \\
\text { Discovery }\end{array}$ & $\begin{array}{l}\text { Resource } \\
\text { Reservatio } \\
\text { n }\end{array}$ & \begin{tabular}{|l|} 
Route \\
Break \\
Prediction
\end{tabular} & \begin{tabular}{|l|} 
Redundant \\
Routes
\end{tabular} & $\begin{array}{l}\text { Network } \\
\text { Architecture }\end{array}$ \\
\hline DSDV & Delay & Yes & Proactive & Yes & Yes & No & Flat \\
\hline CEDAR & Bandwidth & No & $\begin{array}{l}\text { Proactive } \\
\text { /Reactive }\end{array}$ & Yes & No & No & Hierarchical \\
\hline $\begin{array}{l}\text { TICKET- } \\
\text { BASED }\end{array}$ & $\begin{array}{l}\text { Bandwidth } \\
\text { Delay }\end{array}$ & No & Proactive & Yes & No & Yes & Flat \\
\hline OLSR & Bandwidth & Yes & Proactive & No & No & No & Hierarchical \\
\hline AQOR & $\begin{array}{l}\text { Bandwidth } \\
\text { Delay }\end{array}$ & Yes & Reactive & Yes & No & No & Flat \\
\hline ADQR & Bandwidth & No & Reactive & Yes & Yes & Yes & Flat \\
\hline TDR & Bandwidth & No & Reactive & Yes & Yes & No & Flat \\
\hline BEQR & Bandwidth & Yes & Reactive & No & No & No & Flat \\
\hline WRP & Bandwidth & Yes & Proactive & No & Yes & Yes & Flat \\
\hline
\end{tabular}

Table 1 Comparison of Routing Protocols

VII.CONCLUSION

In this paper a comparison of various routing protocols in terms of their support for node mobility, routing overhead, design issues and support for scaling of a network. It also focuses open issues that must be addressed in QoS aware routing in terms of bandwidth, delay estimation, route discovery, resource reservation and route maintenance. This paper also focuses on various quality of service improving methods in MANET's in terms of routing such as enhancing the routing technique use of dynamic queue length; find the optimal path from multiple paths, to find multiple node disjoint routes, selection of routes based on quality of service metrics. This is also contains comparison of all these methods based on parameters such as routing overhead, protocol is loop free or not, it supports multiple paths or not and the protocols are proactive, reactive or hybrid. Hence overall these methods help to improve design issues and performance MANET's.

\section{REFERENCES}

[1] Mrs. Manisha P. Navale, Prof. G.T. Chavan, "Survey on QoS improving method in MANET,", IJERT, Vol.3 Issue 12, Dec, 2014.

[2] Lie Chen and Wendi B. Heinzelman, " A Survey of Routing Protocols that Support QoS in Mobile Ad Hoc Networks," IEEE Network, Nov-Dec, 2007

[3] Prasant Mohapatra, Jian Li and Chao Gui,"QoS in Mobile Ad Hoc Networks," IEEE Wireless Communications, June2003.

[4] Ashraf Abu-Ein, Jihad Nader, "An enhanced AODV routing protocol for MANETs", IJCSI International Journal of Computer Science Issues, Vol. 11, Issue 1, No 1, January 2014.

[5] Mamata Balachandra, Prema K V, Krishnamoorthy M, "Enhancing the Quality of Service in MANET by Improving the Routing Technique", International Journal of Computer Applications Volume 51-No.7, 2012.

[6] C.Siva Ram Murthy,B.S.Manoj "Ad Hoc Wireless Networks architectures and protocols," book, pearson educaton .

[7] A.Cacciapuoti, M.Caleffi, and L.Paura, "Reactive routing for mobile cognitive radio ad hoc networks," Ad Hoc Netw., vol. 10, no. 5, pp. 803-815, Jul. 2012.

[8]Sinha, R. Sivakumar, and V. Bharghavan, "CEDAR: A CoreExtraction Distributed Ad hoc Routing Algorithm," IEEE INFOCOM '99, New York, NY,Mar. 1999.

[9] Karthikeyan, D.; Dharmalingam, M., "Ant based intelligent routing protocol for MANET," in Pattern Recognition, Informatics and Mobile Engineering (PRIME), 2013 International Conference on, vol., no., pp.11-16, 21-22 Feb. 2013

[10] RameshPalanisamy, V. Mathivanan "Efficient Zone Based Routing Protocol (EZBRP)" International Journal of Computer Scienceand Information Security (IJCSIS), Vol. 14, No. 5, May 2016.

[11] Q. Xue and A. Ganz, "Ad Hoc QoS On-demand Routing (AQOR) in MobileAd hoc Networks," J. Parallel and Distrib. Comp., vol. 62, no. 2, Feb.2003, pp. 154-65. 